\title{
Switching off MDSCs by targeting REL
}

REL (also known as c-Rel) is a member of the nuclear factor- $\kappa \mathrm{B}$ (NF- $\kappa \mathrm{B})$

family of transcription factors that is expressed in both myeloid and lymphoid cells. Although it has been shown that loss of REL in lymphoid cells (specifically regulatory $\mathrm{T}\left(\mathrm{T}_{\text {reg }}\right)$ cells) can inhibit tumour growth, its role in myeloid cells is not clear. Ting Li, Xinyuan Li et al. explored the role of REL in tumour growth in more detail and determined that REL has a key role in generating myeloid-derived suppressor cells (MDSCs) and that targeting REL might be used for cancer immunotherapy.

The authors first subcutaneously injected mouse melanoma (B16F0 or B16F10) or lymphoma (EL4) cells into either wild-type or $\mathrm{Rel}^{-/-}$mice. They observed a marked reduction in tumour size and weight in $\mathrm{Rel}^{-/-}$mice. In order to ascertain the roles of REL in different cell types during tumour development, they depleted either $\mathrm{T}_{\text {reg }}$ cells (CD25+ cells) or Gr- $1^{+}$myeloid cells (which include MDSCs) and found that in wild-type mice, Gr- $1^{+}$cell depletion blocked B16F10 tumour growth more than $\mathrm{CD} 25^{+}$cell depletion did. Knockout of Rel selectively in $\mathrm{Gr}-1^{+}$cells also significantly reduced B16F10 tumour growth, indicating the importance of REL in this phenomenon.

B16F10 tumour-bearing mice lacking Rel globally or in $\mathrm{Gr}-1^{+}$cells had reduced numbers of MDSCs (defined as $\mathrm{Cd} 11 \mathrm{~b}^{+} \mathrm{Gr}-1^{+}$cells) both in the blood and within tumours. Tumour-naive mice lacking $R e l$ in Gr- $1^{+}$cells did not have alterations in the levels of various myeloid cells (including Cd $11 \mathrm{~b}^{+} \mathrm{Gr}-1^{+}$cells) or lymphoid cells, which suggests a role for REL specifically in tumour-bearing mice.

B16F10 tumours that formed in mice lacking $\mathrm{Rel}$ in $\mathrm{Gr}-1^{+}$cells had increased infiltration of CD8 ${ }^{+}$ effector T cells. Furthermore, co-culture of $\mathrm{Cd} 11 \mathrm{~b}^{+} \mathrm{Gr}-1^{+}$cells isolated from tumour-bearing
$\mathrm{Re}^{-1-}$ or wild-type mice with $\mathrm{CD}^{+}$

$\mathrm{T}$ cells from wild-type mice indicated that the MDSCs from $\mathrm{Rel}^{-1-}$ mice were not as effective in suppressing the activity of $\mathrm{CD}^{+} \mathrm{T}$ cells. This functional defect of $\mathrm{Cd} 11 \mathrm{~b}^{+} \mathrm{Gr}-1^{+}$ cells from $\mathrm{Rel}^{-1-}$ mice was rescued by re-expressing Rel.

To analyse the differences between MDSCs isolated from wild-type and $\mathrm{Rel}^{-1-}$ mice, the authors conducted RNA sequencing. Many pro-tumoural genes (such as Arg1, Cebpb and many genes encoding proteins involved in metabolic pathways) were downregulated in $\mathrm{Rel}^{-1-}$ MDSCs, and many anti-tumoural, inflammatory genes (such as Tnf and Il1b) were upregulated in these cells. These results were confirmed by reverse transcription PCR and western blotting to look at protein levels.

REL was able to bind to and activate promoter regions of $A r g 1$ and $C e b p b$ during MDSC development (stimulated by granulocyte-macrophage colony-stimulating factor (GM-CSF)). Additional transcription factors $(\mathrm{C} / \mathrm{EBP} \beta$ (which is encoded by Cebpb), STAT3 and p65 (also known as RELA)) also bind to these promotors and seem to form an enhanceosome complex nucleated by REL.

Increased anti-tumoural inflammatory gene expression in $\mathrm{Rel}^{-1-}$ mice was likely due to the reduced expression of $\mathrm{C} / \mathrm{EBP} \beta$, as re-expressing Cebpb in $\mathrm{Rel}^{-1-}$ MDSCs suppressed the expression of these genes. Re-expression of $C e b p b$ also restored the ability of these MDSCs to suppress $\mathrm{CD}^{+} \mathrm{T}$ cell functions.

The authors had previously developed a pharmacological inhibitor of REL (R96A) and based on the above results hypothesized that it might have potential as a cancer therapy. Several lines of evidence confirmed the specificity of R96A for REL. When injected into wild-type mice at the time of tumour cell implantation and then daily throughout the duration of the experiments, R96A reduced

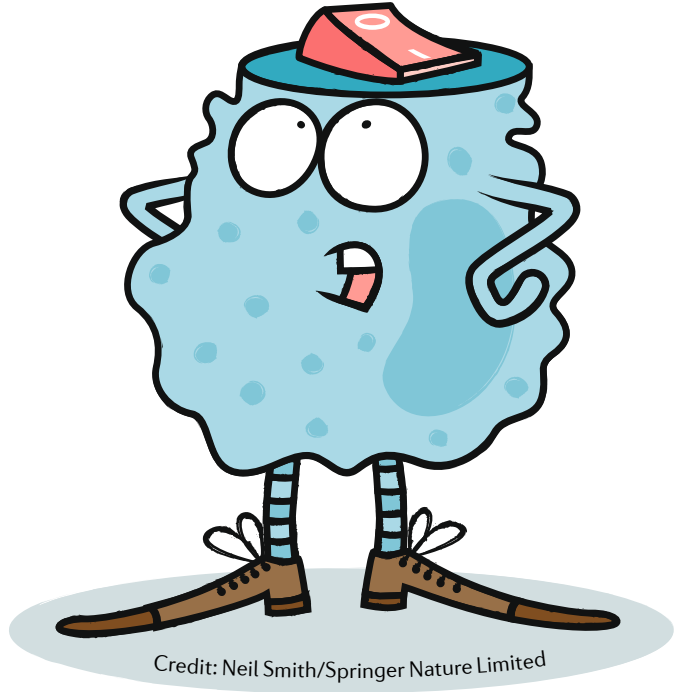

the growth of B16F10 and EL4 tumours. Growth of B16F10 cells in $\mathrm{Rel}^{-1-}$ mice was unaffected by R96A. Consistent with its anti-tumour effects, R96A also blocked MDSC development and function in mice and in culture. Furthermore, a combination of R96A and a T cell checkpoint inhibitor (anti-programmed cell death protein 1 (PD1)) administered at the time of B16F10 cell implantation more strongly prevented tumour growth than either R96A or anti-PD1 alone.

To determine the relevance of this pathway in human cells, the authors cultured human peripheral blood mononuclear cells in the presence or absence of R96A and stimulated them with GM-CSF and IL- 6 to induce MDSC generation. MDSCs generated in the presence of REL inhibition had strongly reduced suppressive activities against $\mathrm{CD} 8^{+} \mathrm{T}$ cells and reduced expression of $C E B P B$ and $A R G 1$ mRNA, mirroring the results observed in mice.

These results suggest the importance of a REL-controlled pathway driving MDSC development and inhibiting anti-tumour immunity. Further work will need to be done to determine whether inhibition of REL by R96A or other inhibitors can be used therapeutically to reduce the size of, or eliminate,
$\mathrm{R} 96 \mathrm{~A}$ reduced the growth of B16F10 and EL4 tumours
Sarah Seton-Rogers

ORIGINAL ARTICLE Li, T. et al. c-Rel is a myeloid established tumours in mice and to confirm the relevance of this pathway in patients with cancer. checkpoint for cancer immunotherapy. Nat. Cancer 1, 507-517 (2020) 